\title{
Measuring Curved Crystal Performance for a High-Resolution, Imaging X-Ray Spectrometer
}

\author{
Michael J. Haugh ${ }^{1}$ and Richard Stewart ${ }^{2}$ \\ ${ }^{1}$ National Security Technologies, LLC (NSTec), 161 S. Vasco Road, Livermore, CA 94550, USA \\ ${ }^{2}$ Lawrence Livermore National Laboratory (LLNL), 7000 East Avenue, Livermore, CA 94550, USA \\ Correspondence should be addressed to Michael J. Haugh, haughmj@nv.doe.gov
}

Received 16 December 2009; Accepted 7 June 2010

Academic Editor: Gene Ice

Copyright (C) 2010 M. J. Haugh and R. Stewart. This is an open access article distributed under the Creative Commons Attribution License, which permits unrestricted use, distribution, and reproduction in any medium, provided the original work is properly cited.

\begin{abstract}
This paper describes the design, crystal selection, and crystal testing for a vertical Johann spectrometer operating in the $13 \mathrm{keV}$ range to measure ion Doppler broadening in inertial confinement plasmas. The spectrometer is designed to use thin, curved, mica crystals to achieve a resolving power of $E / \Delta E>2000$. A number of natural mica crystals were screened for flatness and X-ray diffraction width to find samples of sufficient perfection for use in the instrument. Procedures to select and mount high quality mica samples are discussed. A diode-type X-ray source coupled to a dual goniometer arrangement was used to measure the crystal reflectivity curve. A procedure was developed for evaluating the goniometer performance using a set of diffraction grade Si crystals. This goniometer system was invaluable for identifying the best original crystals for further use and developing the techniques to select satisfactory curved crystals for the spectrometer.
\end{abstract}

\section{Introduction}

The analysis of the X-ray emission line spectrum of highly ionized atoms in high temperature inertial confinement target implosions is a powerful tool for the characterization of plasma conditions in the target [1]. In principle, space and time resolved spectroscopic data can be used to determine three dimensional plasma conditions in the compressed plasma. Electron densities can be determined from Stark broadened line profiles and continuum edge shifts, and electron temperatures can be determined from line ratios and continuum distributions [2]. In most current inertial confinement experiments however, ion temperatures have been impossible to measure from X-ray spectroscopy because Stark broadening and collisional broadening of dopant ions are larger than the thermal ion Doppler broadening that could be used to measure ion temperatures. While neutron and proton emission time histories and spectra can provide some data on ion temperatures, no satisfactory technique currently exists to measure time and space resolved ion temperatures in implosion plasmas, and any viable technique for ignition targets will likely have to rely on X-ray emission rather than on neutron or proton emission because of time of flight dispersion of particle arrival times at the detectors as well as proton range effects.

Yaakobi et al. [3, 4] have shown, however, that for very highly charged $\mathrm{Kr}$ XXXV ions in exploding pusher implosions, which achieve ion temperatures in excess of $10 \mathrm{keV}$, ion Doppler broadening in low lying helium like 13-15 keV resonance lines may be observable if spectra can be recorded with sufficiently high spectral resolution. This is basically possible because the energy levels of the highly charged krypton ions are tightly bound, have large relativistic fine structure splitting, have low polarization in the plasma microfield, and have small collision cross sections. The observable lines least sensitive to Stark broadening are the $1 \mathrm{~s}$ to $2 \mathrm{p}$ resonance transitions at $13029 \mathrm{eV}$ and $13115 \mathrm{eV}$. Similar conditions are expected in experiments that will be possible when the ignition of thermonuclear burn is achieved with the next generation of megajoule class lasers like NIF, where nuclear energy released is expected to produce electron temperatures as high as $50 \mathrm{keV}$ and even higher ion temperatures. The high electron temperatures will produce hydrogen and helium like ions in heavy dopant 


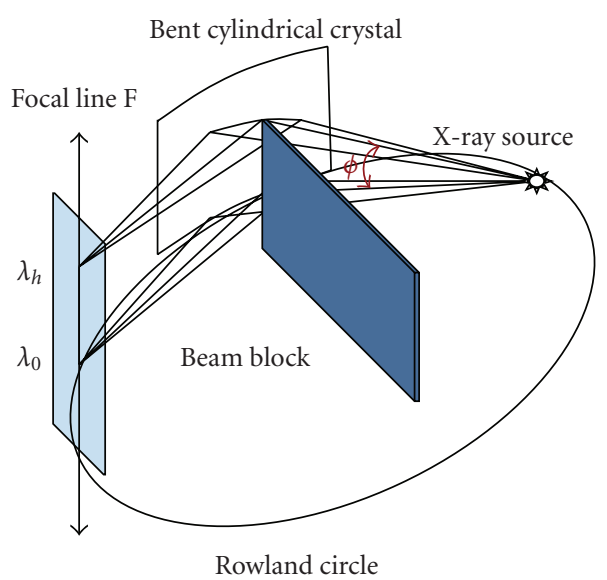

(a)

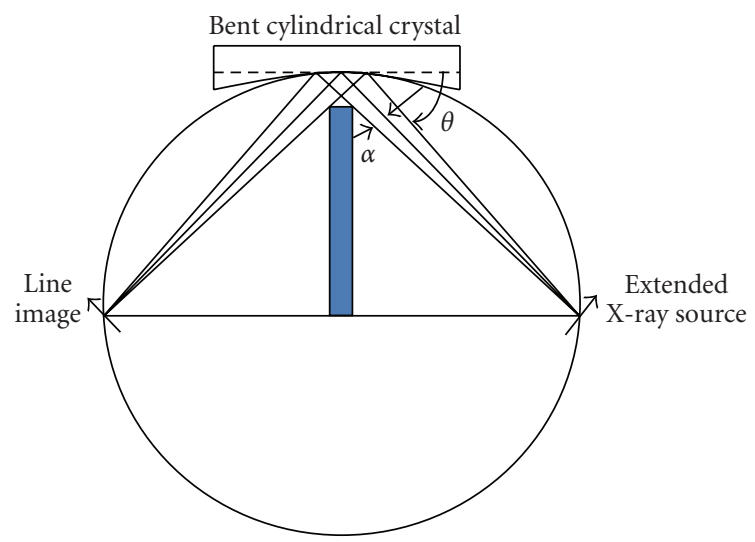

(b)

Figure 1: (a) Vertical Johann Spectrometer Scheme-Layout (b) Vertical Johann Spectrometer Scheme-Top view looking down cylinder axis.

atoms such as krypton and xenon, and the broadening of $1 s$ to $2 p$ resonance lines from these ions is predicted to be dominated by ion-Doppler broadening despite the very high expected plasma electron densities in excess of $10^{26} \mathrm{e}^{-} / \mathrm{cm}^{3}$. The $\mathrm{Kr}$ dopant can be used in exploding pusher gas, targets on NIF, or with high atomic number impurities in the solid portion of cryogenic targets.

The resolving power necessary to measure Doppler broadening in these heavy ions is expected to be $E / \Delta E \geq$ 2000 or greater, depending on the ion temperature achieved and the mass of the ions being observed. This type of measurement is now standard in Tokamak experiments [5], but it is difficult to achieve on laser driven implosions due to the relatively low brightness of the source and the restricted viewing solid angle and space available for instrumentation. In this paper, we describe the design of a small onedimensional imaging spectrometer that is compatible with the experimental space constraints for direct drive laser implosions, and that can achieve high resolving powers for helium-like krypton transitions at $13 \mathrm{keV}$. We also describe our work on the design and characterization of the curved diffraction crystals needed to provide sufficient resolving power for the spectrometer. This type of instrument could be used to make time and space resolved measurements of the ion temperature in exploding pusher krypton doped plasmas or other very high temperature plasma experiments that are expected to be possible in the future.

\section{Spectrometer Design}

The spectrometer we are designing is a version of a vertical Johann spectrometer (VJS). The principle of this type of spectrometer has been described in detail by Renner et al. $[6,7]$ and Kopecký $[8]$.

The main element of the VJS is a cylindrically curved crystal that diffracts X-rays from a source of small spatial extent located on the Rowland circle of the crystal. In a normal Johann spectrometer, X-rays from a monochromatic point source are approximately focused to an opposing point on the Rowland circle in a plane normal to the axis of the cylinder containing the point source, as shown in Figure 1. It is easy to show that this focusing is conserved for points along the line parallel to the cylinder axis that intersects the Rowland circle above this central plane, as shown in Figure 1(a). A point source on the Rowland circle focuses to a line shown as F in Figure 1(a). The angle of incidence of lines incident on the diffraction crystal increases slowly with increasing elevation angle from the Rowland circle, so that the sine of the angle of incidence varies as

$$
\sin (\theta)=\sin \left(\theta_{0}\right) \cos (\phi) .
$$

The wavelength of diffracted rays is determined by the Bragg relation

$$
\lambda=2 d \sin \Theta .
$$

This means that the wavelength of X-rays diffracted from the crystal for the point source varies with $\phi$ as

$$
\lambda_{h}=\lambda_{0} \cos \phi,
$$

where $\lambda_{0}$ is the wavelength focused in the plane of the Rowland circle and $\lambda_{h}$ is the wavelength for the ray tilted at an angle $\phi$ to the plane.

For high resolution, the length of the crystal illuminated by the source must be limited because the angle of incidence on the crystal from a point source slowly changes as the Xrays from the source hit the crystal at increasing distances from its center. This is the familiar Johann defocusing defect. The angular size of the crystal used can be controlled with the beam block shown in Figures 1(a) and 1(b).

For extended sources, the cylindrical crystal focuses the image of the source in one dimension perpendicular to the ray diffracting from the crystal. The high vertical dispersion of the spectrum greatly reduces the effects of source size on spectral resolution in the direction of the axis. For sources displaced from the Rowland circle in the direction perpendicular the ray striking the crystal and the axis of 


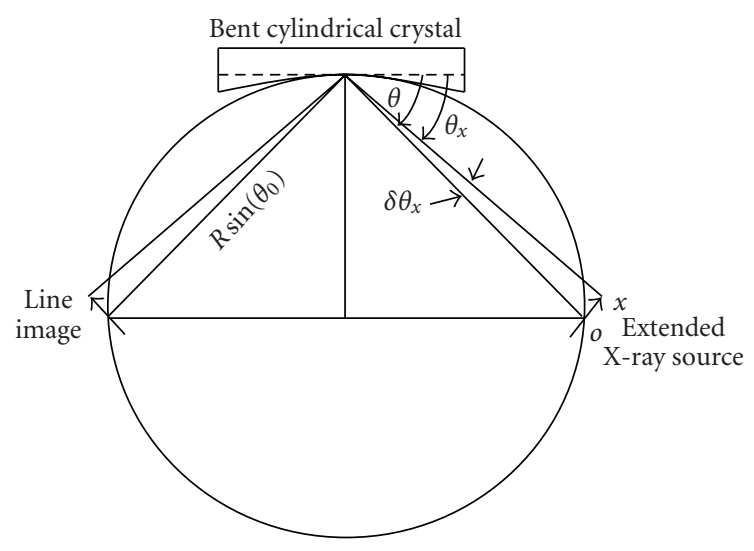

FIGURE 2: Spectral line formation geometry.

symmetry of the crystal, as shown in Figure 2, the spectrum is shifted spectrally relative to the point on the Rowland circle because the angles of incidence of all rays are shifted slightly. A small spatial shift in this direction gives a much larger change in Bragg angles on the surface of the crystal than an equivalent vertical translation of diffracted rays in the image plane, so the wavelength at one value of $\phi$ changes very rapidly with horizontal source size, leading to steeply sloped lines on the detector for a given wavelength.

The dependence of the line position on the position of the source can be calculated from the geometry of the spectrometer. For a distance $x$ shown in Figure 2, displaced laterally from the Rowland circle, the change in Bragg angle in the plane of the Rowland circle is given by (4):

$$
\delta \Theta_{0 x}=\tan ^{-1}\left[\frac{x}{R \sin \Theta_{0}}\right], \quad \Theta_{0 x}=\Theta_{0}-\delta \Theta_{0 x},
$$

where $R$ is the radius of curvature of the crystal.

Then the angle of inclination of the diffracted X-rays of wavelength $\lambda$ for a given point $x$ displaced off the Rowland circle is given by

$$
\phi_{x}=\cos ^{-1}\left[\frac{\lambda}{2 d \sin \Theta_{0 x}}\right] .
$$

The displacement of an X-ray diffracting at $\phi_{x}$ in the vertical (axial) direction of the diffracted line is then given by

$$
z=\frac{2 R \sin \left(\Theta_{0}\right) \tan \left(\phi_{x}\right)}{\cos \left(\delta \Theta_{0 x}\right)}
$$

The one dimensional imaging of the detector allows these spatially dependent spectral profiles to be separated. If a detector with poor or no spatial resolution in the imaging direction is used, the combined spectral effects are equivalent to the normal source size broadening for a flat crystal.

The VJS has several properties that make it useful for laser experiments. It has high collection efficiency due to having the source on the Rowland circle, where all rays at one angle of inclination phi hitting the crystal are collected and focused to a point. It has very high dispersion independent of wavelength and Bragg angle due to vertical dispersion. This means that small Bragg angles and small beam deflections can be used in a very high resolution design, which allows us to fit the spectrometer into a very small solid angle that is compatible with direct drive laser experiments. The large vertical dispersion also allows us to use detectors with relatively poor spatial resolution in one dimension. Finally, the spectrometer has a spatial resolution that can be better than 10 microns in one direction for a properly bent crystal.

For our experiments we have designed a spectrometer to look at the Kr XXXV 1s to $2 \mathrm{p}$ resonance lines. By using only the portion of the crystal diffracting rays at an angle near $13^{\circ}$ angle of inclination and then rotating the source, crystal, and detector around the line normal to the center of the crystal, we can achieve the vertical Johann geometry in a small linear package that can fit in a diagnostic tube. The layout is shown in Figure 3, which shows the schematic design for a vertical Johann instrument designed to be used in a 6 inch diagnostic tube for laser implosion experiments. Bragg angles less than $10^{\circ}$ are required in order to meet the geometric constraints of the diagnostic tube. For our design, the crystal is bent to a radius of $1644 \mathrm{~mm}$, with a $13.4^{\circ}$ angle of inclination, and a Bragg angle of $8.201^{\circ}$ using a mica crystal in third order $(006$ plane).

\section{Crystal Requirements}

The resolution achievable with this spectrometer depends primarily on the diffraction properties of the crystal and the accuracy of its surface alignment to a cylinder. Resolving powers of 2000 to 10000 are required, with higher values allowing lower ion temperatures to be measured and increasing the potential accuracy of the measurement. A variety of bonding techniques can be used to bend the crystal into a cylindrical form with optical precision. We are using fused silica substrates ground to an accuracy of $1 / 4$ wavelength, with a $1642 \mathrm{~mm}$ radius of curvature. The crystals are held between two glass forms with convex and concave curvature and glued with a thin layer of UV curing cement to the concave cylindrical substrate.

Crystal planes that could be used for the $13 \mathrm{keV} \mathrm{X-}$ rays we are measuring, with Bragg angles less than $10^{\circ}$, can have resolving powers $E / \Delta E \sim 10,000$ for flat crystals comprised of low atomic number materials like mica 006, quartz 10-10 and 10-11, and silicon 111. This isotopic approach of Taupin, which depends only on Poisson's ratio and neglects anisotropy of the crystal when bent, agrees well with the Bragg reflections measured by Uschmann et al. $[9,10]$. Unfortunately the diffraction width of these planes is significantly affected by the $1.6 \mathrm{~m}$ radius of curvature being used. The effects of curvature are primarily due to penetration of X-rays close to the Bragg angle at the surface of the crystal far enough into the crystal that the vector normal to the curved lattice planes of the crystal can rotate enough to allow Bragg diffraction. The result of this is a decaying tail of diffraction at lower angles of incidence, dropping slowly from the peak of the pattern. Distortion of the lattice as the X-ray propagates into the crystal is a smaller effect for our conditions. Diffraction patterns for the curved 


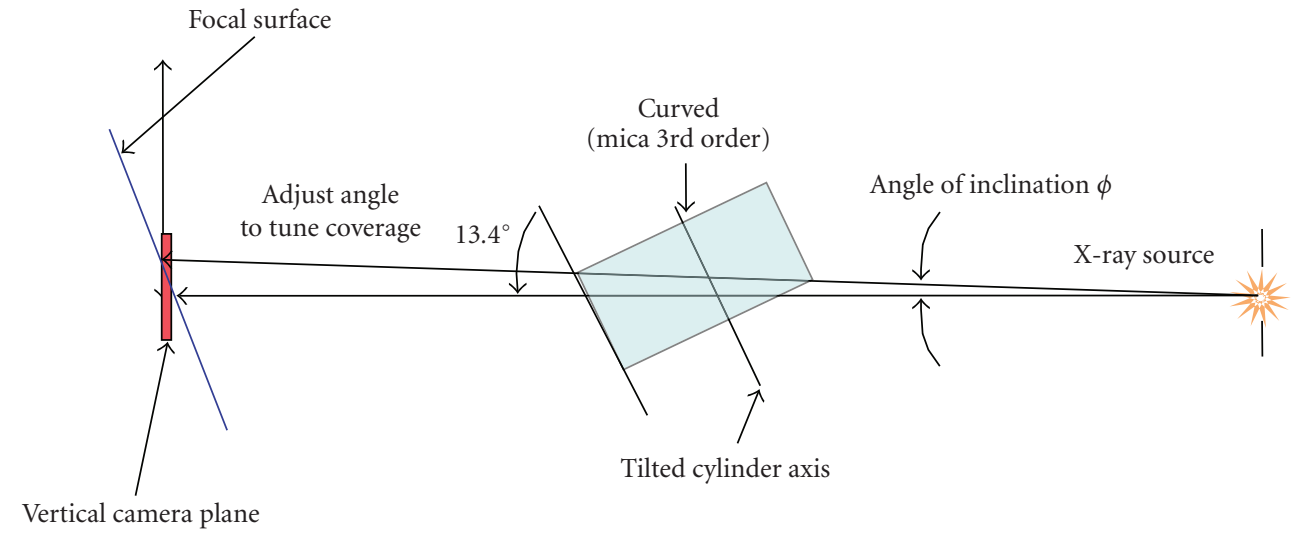

FIGURE 3: Side view of vertical Johann mica spectrometer. X-rays of increasing energy are diffracted at increasing angle of inclination $\phi$.

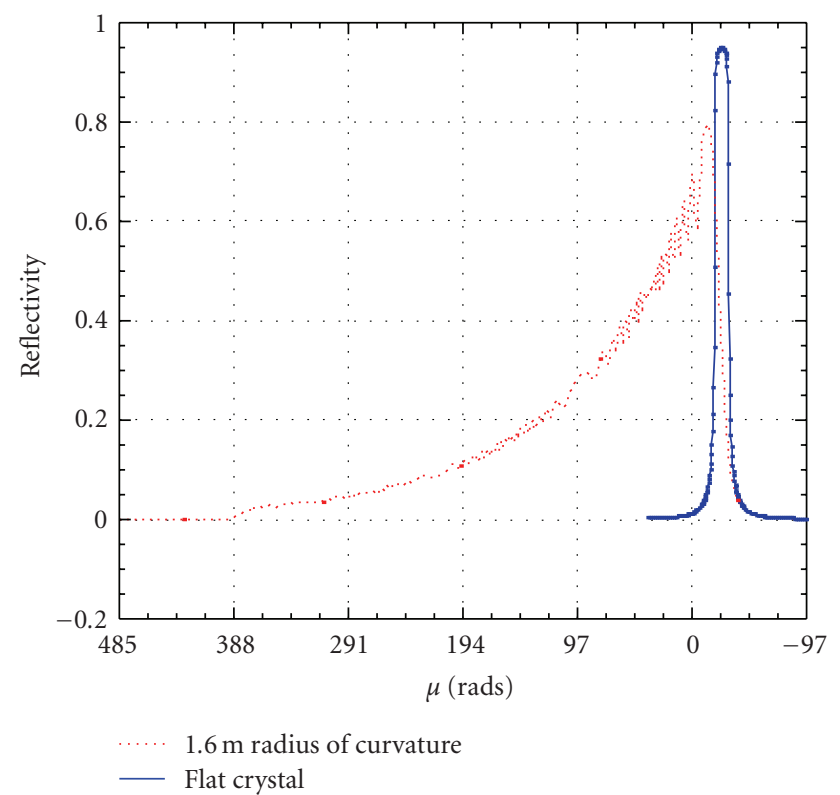

FIGURe 4: Comparison of reflectivity vs angle for a 100 micron thick crystal having a $1.6 \mathrm{~m}$ radius of curvature.

crystals can be calculated from the Takagi-Taupin equations which account for both these effects $[11,12]$.

Figure 4 shows how diffraction pattern calculated with the code from the Taupin equations [9] changes for a flat and 100 micron thick mica 006 (third order reflection) crystal at $13 \mathrm{keV}$. The tail increases the integrated reflection coefficient for the crystal, but increases the width of the curve. It is theoretically possible to reduce this curvatureinduced broadening by using a crystal that is thinner than the propagation depth of the X-rays, as shown in Figure 5. The tail on the diffraction curve is reduced as the crystal thickness is reduced. Five micron thick crystals have curves very similar to the flat crystal. With a 5 micron thick perfect mica crystal of $1.644 \mathrm{~m}$ radius using $6 \mathrm{~mm}$ of arc, ray tracing calculations show that the resolving power possible is about 7800 .

Higher atomic number crystals with higher absorption at $13 \mathrm{keV}$ do not have large changes to the flat crystal diffraction widths due to crystal curvature. This is because their absorption prevents X-rays from reaching deep enough into the crystal to form large diffraction tails. Germanium 111 is an example of this type of crystal, which can have a resolving power of 3160 at $13 \mathrm{keV}$. This crystal has less resolution than a perfect thin mica crystal, but has the advantages of higher integrated reflection, consistently more perfect grown crystals, and surfaces that can be polished optically flat. Ge 111 crystals were mounted and tested in the same way as mica, so they could be used for initial testing and as a backup for the mica crystal.

\section{Monochromator Crystal Selection}

Mica (006) and Si (111) have nearly the same reflectivity as flat, perfect crystals. But mica has been selected as the best candidate for the VJS because it can be cleaved to the 5 micron thickness and bent to the desired curvature. This is not practical for $\mathrm{Si}$ (111) and quartz. Si (111) crystals are used to evaluate the dual goniometer system performance. Dicing Si or quartz crystals was considered but rejected as being too costly.

Manufacturing crystals thinner than $100 \mu \mathrm{m}$ that have dimensions on the order of $1 \mathrm{~cm}$ in the crystal axis orientation needed is very difficult for most available crystals except for mica. Mica has very weak binding between planes, and is readily cleaved to thickness of 1 micron or even thinner. Mica tends to be an imperfect crystal, however, with distorted surfaces and mosaic broadening occurring in most crystals. But studies done by Uschmann et al. [10] have shown that some crystals can be flat and perfect on an atomic scale over areas of up to $20 \mathrm{~cm}^{2}$.

Natural mica crystals vary considerably in their diffraction quality. Mica crystals were purchased from a variety of sources, and a synthetic crystal was also tested. The crystals were tested flat in the first stage. The procedure for evaluating these crystals began with optical testing, selection of those having the best quality, and measurement of the X-ray reflectivity curve for the selected crystals. We have examined several hundred crystals visually and interferometrically using Fizeau and Haidinger fringes with optical flats in 

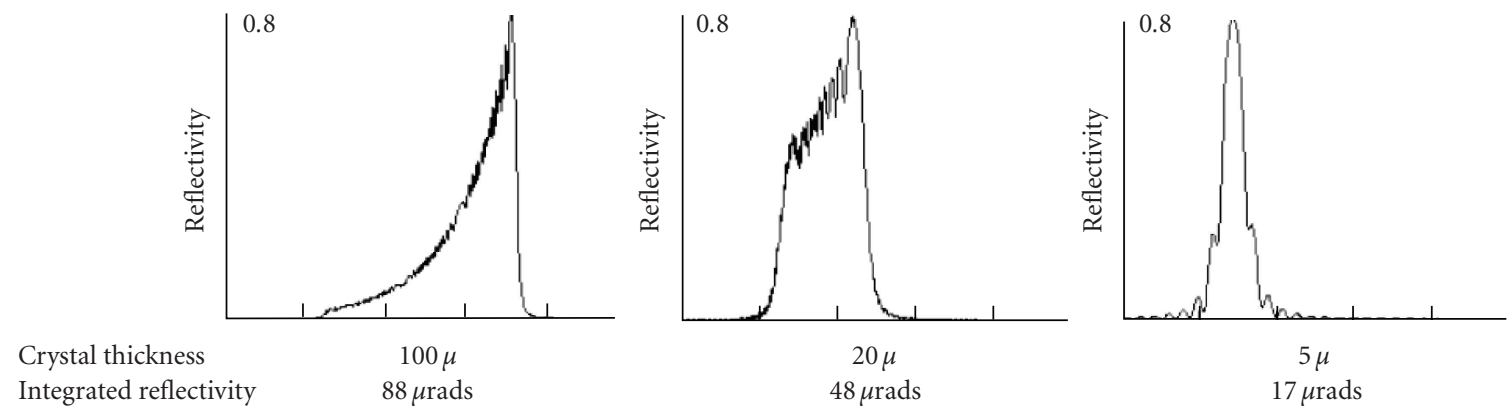

FIGURE 5: Broadening due to curved crystal curvature with crystals of thickness varying from 5 microns to 100 microns at $1.6 \mathrm{~m}$ radius of curvature, calculated with Takagi-Taupin equation.

TABle 1: Crystals Used, the Miller Indices, and the Bragg Angle at $11442.3 \mathrm{eV}$.

\begin{tabular}{lccc}
\hline Crystal & Silicon 111 & Mica 006 & Germanium 111 \\
\hline Bragg angle, degrees & 9.950 & 9.347 & 9.547 \\
\hline
\end{tabular}

monochromatic light to find sufficiently perfect mica crystals for our needs. The crystals were examined as 200 to $300 \mu \mathrm{m}$ thick slabs. Relatively few were acceptable for the X-ray testing, and this became a learning process for the best sources of mica crystals.

The crystals that passed the optical evaluation were then tested using X-rays as thick flats. There were many more rejections in this phase. The best flat candidates were mounted at their original thickness with UV curing glue on fused silica curved substrates, and then cleaved in water to thicknesses of 3-10 microns while attached to the substrate. We were unable to first cleave the crystals and then mount them, as they wrinkled uncontrollably when they contacted the UV cement. The reflectivity curve for these curved crystals was then measured.

\section{Characterizing the $X$-ray Diffraction}

Measurements of X-ray reflectivity for mica and Ge crystals were done using the $A u L_{\beta 1}$ spectral line at $11442.3 \mathrm{eV}$. Measurements on the Si crystal were done using the $\mathrm{Au}$ $\mathrm{L}_{\beta 1}$ spectral line and the $\mathrm{Au} \mathrm{L}_{\alpha 1}$ spectral line at $9713 \mathrm{eV}$. The Bragg angle at $11443 \mathrm{eV}$ for these crystals is given in Table 1 . The calculated dynamic diffraction properties for a mica crystal at the X-ray energy used for testing are given in Table 2 [13]. All measurements are compared to these quantities. The curve quality needed for high resolution spectroscopy is the width of the reflectivity curve. The Full Width at Half Maximum (FWHM) is an adequate measure of this quality. This width represents an energy spread of the reflected radiation for a given Bragg angle.

The energy spread can be adequately estimated using the differential form of Bragg's Law:

$$
\frac{\Delta E}{E}=\Delta \Theta \cdot \cot \Theta_{B}
$$

where $E$ is the band center of the spectral line, $\Delta E$ is the energy spread produced by the crystal reflectivity width, $\Delta \Theta$ is the FWHM for the reflectivity curve, $\Theta_{B}$ is the Bragg angle.
For the conditions in Table 2, and assuming an equal polarization mixture so that $\Delta \Theta=12.373 \mu \mathrm{rad}, \Delta E=$ $0.860 \mathrm{eV}$ at $11442.3 \mathrm{eV}$.

5.1. The Dual Goniometer Instrumentation and Technique. The X-ray source used for measuring the mica crystal diffraction performance is a diode type with a water cooled anode. It operates in the medium X-ray energy range, $600 \mathrm{eV}$ to $16 \mathrm{keV}$ and is illustrated in Figure 6 . The source chamber and the sample chamber have independent vacuum controls and are separated by a tubing section that includes a remotely operated valve. The source maximum operating voltage and power are $20 \mathrm{kV}$ and $4000 \mathrm{~W}$. The operating anode was gold for the mica measurements, and the $\mathrm{Au} \mathrm{L}_{\beta 1}$ spectral line was selected.

To collimate the X-ray beam, the sample chamber has a pair of slit mechanisms separated by $200 \mathrm{~mm}$. Each slit mechanism has a vertical and a horizontal slit pair, with the horizontal slit pair operated manually, and the vertical slit pair operated using a computerized motion controller. Both slit opening and slit offset can be operated remotely. The slit mechanism locations are shown in Figure 6.

The dual goniometer system has four separate rotation stages, two for the monochromator crystal and two for the sample crystal stage. The sample stage has three orthogonal translation degrees. Each of these stages has an independent motion control. They are computer controlled and can be programmed for automatic scanning. Rotation precision is $1 \mathrm{mdeg}$, and the translation precision is $1 \mu \mathrm{m}$. The absolute rotation accuracy is determined by the actual alignment of the elements of each rotation stage. A crystal can be moved in the horizontal and vertical directions using the translation stages to produce reflectivity curves so that uniformity of the crystal across the entire surface can be verified.

The crystal arrangement for these experiments is shown in Figure 7. A vacuum compatible, solid state, energy dispersive detector is mounted on the 4th rotation stage. It provides photon counting sensitivity, and the energy 
TAble 2: Calculated Mica 006 Crystal Properties at $11442.3 \mathrm{eV}$.

\begin{tabular}{|c|c|c|c|c|c|}
\hline Polarization & $R_{p}$ & FWHM, mdeg & FWHM, $\mu \mathrm{rad}$ & $\begin{array}{l}\text { Integrated Reflection } \\
\text { Curve, mdeg }\end{array}$ & $\begin{array}{c}\text { Integrated Reflection } \\
\text { Curve, } \mu \mathrm{rad}\end{array}$ \\
\hline \multicolumn{6}{|l|}{ mica 006} \\
\hline$\sigma$ & 0.932 & 0.728 & 12.708 & 0.868 & 15.142 \\
\hline$\pi$ & 0.928 & 0.690 & 12.037 & 0.820 & 14.308 \\
\hline mixed & 0.930 & 0.704 & 12.37 & 0.844 & 14.716 \\
\hline
\end{tabular}

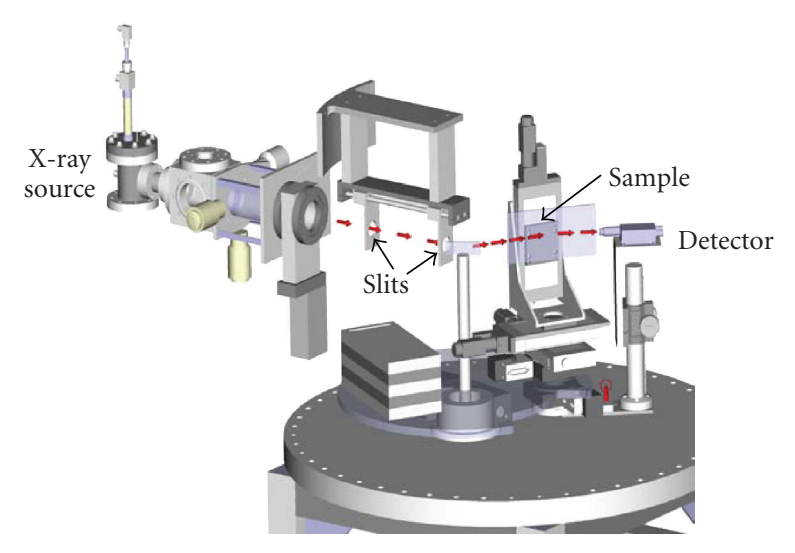

- Beam arrangement

- Dual goniometer

- Sample translation in 3 directions

FIgURE 6: The NSTec X-ray Source and Dual Goniometer for Crystal Measurements, showing the X-ray Beam Path.

information helps to verify that the reflection being observed is in the correct spectral range (i.e., the correct spectral line from the monochromator has been selected, etc.). The X-ray source is $600 \mathrm{~mm}$ from the first slit. The slits are separated by $200 \mathrm{~mm}$. The slit opening for all measurements given in the following sections was $200 \mu \mathrm{m}$. Measurements made using smaller slits did not change the measured reflectivity curve.

5.2. Alignment Procedure. For reliable reflectivity curves, the monochromator crystal and the sample crystal must be properly aligned to the $\mathrm{X}$-ray axis (the rotation axis of the crystal is perpendicular to the $\mathrm{X}$-ray beam axis). They must also be parallel to each other. The system is set up so that a laser beam that can be injected into the X-ray beam path and follow it exactly. The initial system alignment assures that this beam path goes directly over the rotation axis of the monochromator crystal and the sample crystal.

The crystal alignment is done using the laser beam. The monochromator crystal is removed from the system for the sample alignment, and the alignment technique utilizes retro-reflection of the laser beam. The mounting devices for the crystals provide micrometer positioners for precise vertical alignment. The sample crystal is adjusted vertically after rotating the stage so that the crystal faces the slits. Using this micro-positioner, the sample crystal is rotated until the laser beam is reflected exactly back on the slits. The rotation

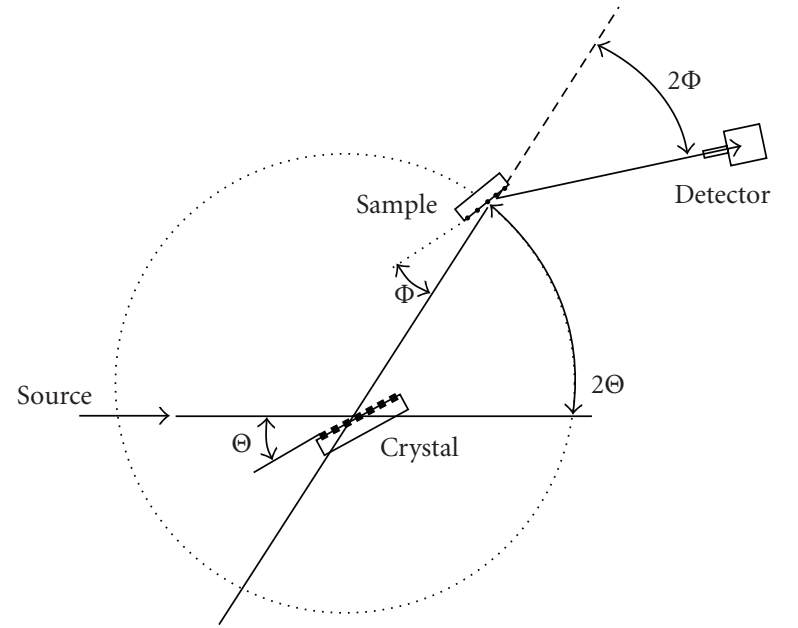

Figure 7: The NSTec X-ray Source and Goniometer for Crystal Measurements, showing Angles.

origin is also obtained by rotating the sample until the reflected laser beam falls unto the slit. The monochromator crystal is then put in place and the same retro-reflection procedure follows, ensuring that the two crystals are parallel.

The system is not designed or operated to measure crystal angles from the goniometer angles exactly. The absolute angle is determined from the spectral analysis and the literature values of the Bragg angle. The relative angles are determined accurately by the goniometers.

\subsection{Measuring the Crystal Reflectivity Curve and Instrumental} Contributions-The Integrated Reflection Curve (IRC) and $F W H M^{*} R_{P}$. Several factors, both instrumental and spectral, influence the measurement of the crystal reflection curve. These factors include the horizontal and vertical beam divergence, spectral energy spread, alignment of the monochromator and sample crystals, and the monochromator crystal reflectivity FWHM. The system performance was evaluated using a set of three diffraction grade silicon crystals. One crystal would be used as a monochromator and the other as a sample crystal. The reflectivity curve of the sample was then measured. The three crystals were interchanged to test all combinations. The reflectivity curves for all combinations agreed within experimental uncertainty, indicating that all three crystals had similar diffraction quality. Typical reflectivity curves for two energies are shown in Figures 8 and 9. 
TABLE 3: Calculated and measured silicon 111 crystal properties at $11442.3 \mathrm{eV}$ and $9713.3 \mathrm{eV}$.

\begin{tabular}{lccccc}
\hline & Peak reflectivity, $R_{p}$ & FWHM, mdeg & FWHM, $\mu$ rad & $\begin{array}{c}\text { Integrated reflection } \\
\text { coefficient, mdeg }\end{array}$ & $\begin{array}{c}\text { Integrated reflection } \\
\text { coefficient, } \mu \text { rad }\end{array}$ \\
\hline $11443.2 \mathrm{eV}$ & 0.966 & 1.280 & 22.344 & 1.571 & 27.42 \\
$\begin{array}{l}\text { Mixed polarization } \\
\text { Measured double crystal }\end{array}$ & 0.41 & 4.1 & 71.6 & 2.13 & 37.2 \\
$\begin{array}{l}\text { Single crystal (calculated from } \\
\text { the double crystal) }\end{array}$ & & 2.85 & 49.7 & & \\
$\begin{array}{l}\text { 9713.3 eV } \\
\text { Mixed polarization }\end{array}$ & 0.953 & 1.504 & 25.250 & 1.820 & 31.76 \\
$\begin{array}{l}\text { Measured double crystal } \\
\begin{array}{l}\text { Single crystal (calculated from } \\
\text { the double crystal) }\end{array}\end{array}$ & 0.35 & 5.2 & 90.8 & 2.31 & 40.3 \\
\hline
\end{tabular}

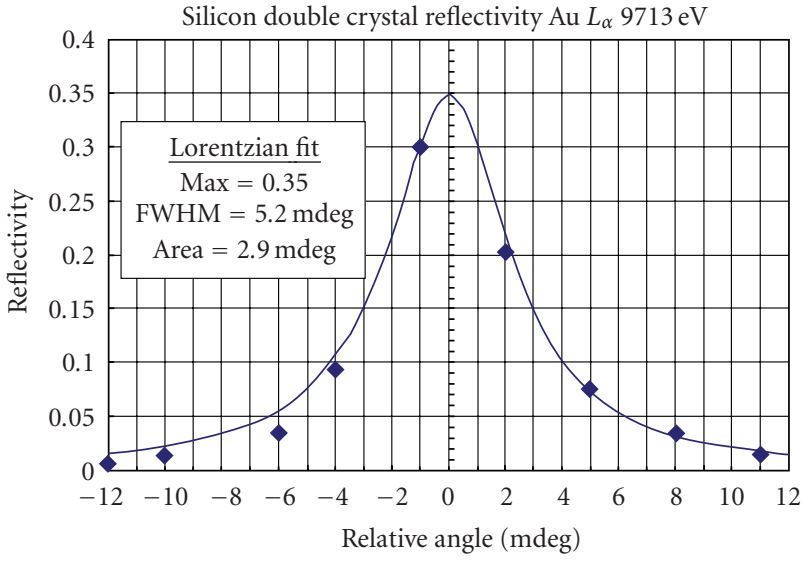

- Data

— Lorentzian

FIGURE 8: Reflectivity curve for Si-Si double crystal measurement at $9713 \mathrm{eV}$.

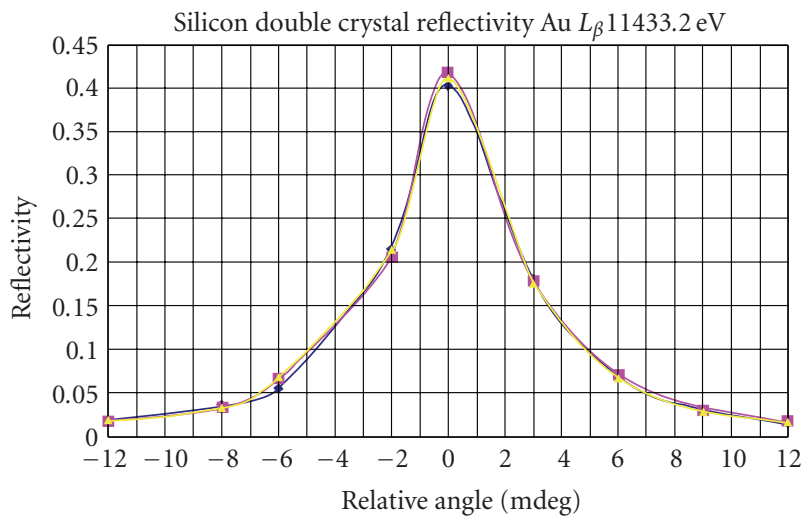

FIguRE 9: Silicon double crystal reflectivity curve from three measurements (shown with three different, closely overlapping, colored curves) taken using the $\mathrm{Au} L_{\beta 1}$ spectral line, $11443.2 \mathrm{eV}$.

These figures show the typical results for all crystal combinations and quite reproducible. Measurements have been done by different instrument operators and separated by extended periods so that the full alignment procedure could be done by the different operators. The motion control system limits the step size to $3 \mathrm{mdeg}$, thus reducing the accuracy of measuring the integrated reflectivity. Figure 8 shows a Lorentzian fit to the data.

The calculated properties for Si 111 are given in Table 3. The measured values are included in the table so that they can be compared easily. A significant feature for this instrument evaluation is the measured FWHM compared to the theoretical. Since this is a two-crystal measurement, the results are increased roughly by $\sqrt{2}$ [9]. This value for FWHM is shown in the row called Single Crystal, and is a factor of about 2.2 larger than the value expected theoretically. The integrated reflectivity is larger than the theoretical value. The uncertainty in the FWHM is on the order of $20 \%$ and that of the integrated reflectivity is $30 \%$ based on the reproducibility of the measurements. Since the two crystals are the same, they have the same diffracting plane separation and the same Bragg angle, so the measurements should be unaffected by the Au spectral band width of $8 \mathrm{eV}$. The $4 \mathrm{mdeg}\left(0.004^{\circ}\right)$ reflectivity curve width calculated from (6) corresponds to $5 \mathrm{eV}$.

These results imply that there is an instrument contribution to the measurement of the FWHM. It was not deemed practical or necessary to determine the origin of this instrument contribution. It has been discussed in the literature $[14,15]$ that the integrated reflectivity is not sensitive to instrument parameters. The purpose of the reflectivity measurements is to select the best mica crystals. Since the integrated reflectivity is less influenced by the instrument properties than the peak reflectivity $\left(R_{P}\right)$ and the FWHM, it was chosen for this comparison.

\section{Measured Crystal Diffraction Properties for Mica and Germanium}

As discussed in the previous section, the goniometer system produced an instrumental broadening of the Si reflectivity curve. Since the integrated reflection curve is not influenced by these instrument parameters, it was chosen to be the measure used to evaluate the mica crystal quality. All measurements were done using an Si monochromator, the same crystal that was used for the measurements shown in 


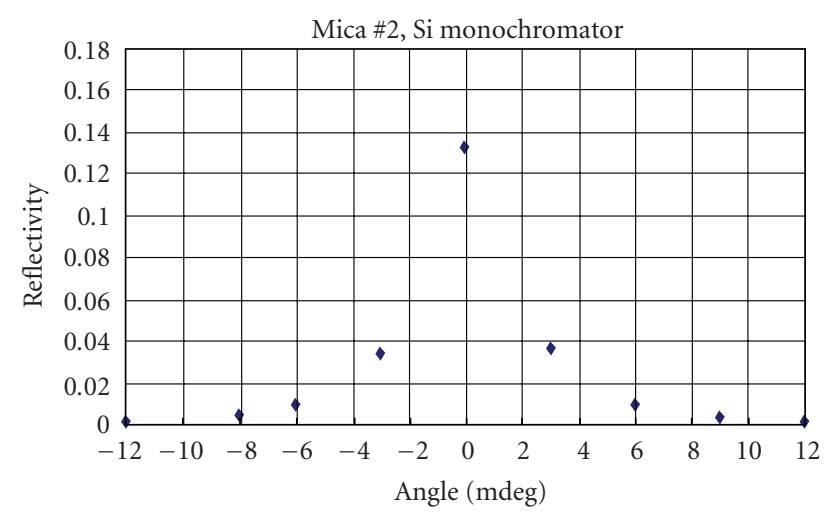

\begin{tabular}{|l|c|c|}
\hline & Measured & Calculated \\
\hline$R_{p}$ & 0.17 & 0.93 \\
\hline FWHM (mdeg) & 4 & 0.7 \\
\hline $\begin{array}{c}\text { Integrated reflection } \\
\text { curve (mdeg) }\end{array}$ & 0.84 & 0.84 \\
\hline
\end{tabular}

FIGURE 10: Reflectivity curve for the best mica flat crystals, $300 \mu \mathrm{m}$ thick.

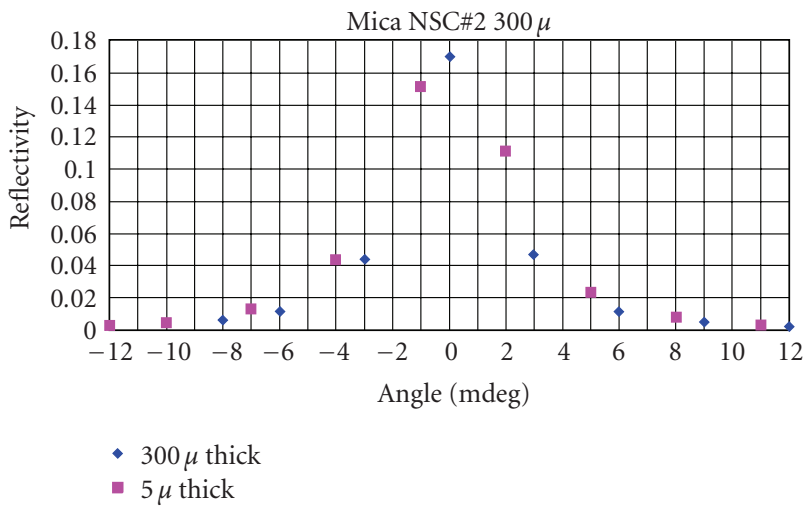

FIGURE 11: Comparing the crystal shown in Figure 10 when it was $300 \mu \mathrm{m}$ thick to the same crystal after it was cleaved to $5 \mu \mathrm{m}$ thick.

Figures 8 and 9. A round robin comparison of the three $\mathrm{Si}$ crystals all gave reflectivity curves similar to Figure 9. The Xray illuminated area was near $1.5 \mathrm{~mm}$ high by $0.30 \mathrm{~mm}$ wide. For the better crystals, several points were measured in the $1 \mathrm{~cm}^{2}$ area that would be illuminated in the VJS.

The initial measurements were done on two square centimeter mica crystals about $300 \mu \mathrm{m}$ thick attached to an optically flat glass substrate. The first set of mica crystals fell into two categories. The majority had an integrated reflection curve (IRC) that was significantly larger than the calculated value given in Table 2. There were a few that measured an IRC that was within experimental uncertainty of the theoretical value but the FWHM was near 10 mdeg and so was larger than the instrumental contribution observed in the Si double crystal measurements. Then we came upon the first crystal that matched the theoretical IRC and the FWHM was at the instrumental limit that was obtained with the Si double crystal measurement. This is shown in Figure 10.

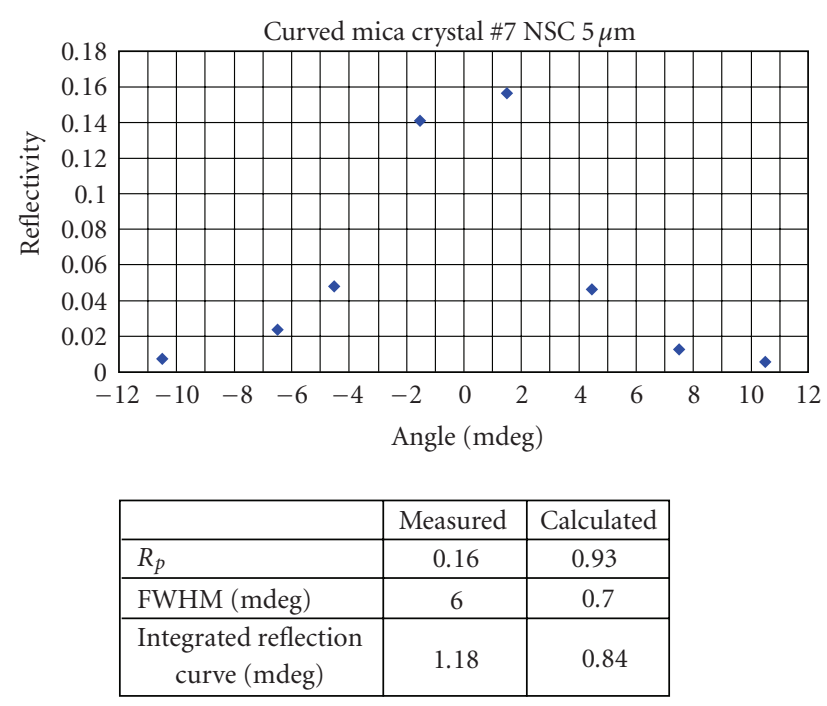

FIGURE 12: Typical best reflectivity curve for good quality mica attached properly to a curved glass substrate.

It appears that mica IRC values can match the theoretical value with diffraction widths at the apparent instrumental limit. Therefore, the criterion for our decision that a mica crystal might have sufficient diffraction quality for the high resolution spectrometer was that the FWHM be near 4 mdeg $\left(0.004^{\circ}\right)$.

The flat crystal whose measurement is shown in Figure 10 was then cleaved to a thickness of $5 \mu \mathrm{m}$ while still glued to the flat glass substrate, and the reflectivity curve was measured again. The result of this measurement is shown in Figure 11 along with the data from the thick crystal. Within the measurement uncertainty, these diffraction curves are identical. The cleaving did not disturb the crystal diffraction quality. At $9.4^{\circ}$ the path through the mica is much larger than the mica extinction length $(2.6 \mu \mathrm{m})$.

The next step was to take crystals that had come from sources that provided the satisfactory flat crystals and test them for X-ray diffraction quality. The $300 \mu \mathrm{m}$ thick mica flat was glued to a glass substrate that was curved in one direction to a $1.6 \mathrm{~m}$ curvature radius and flat in the other direction. The mica was then cleaved to a thickness of $5 \mu \mathrm{m}$. This procedure took some practice to develop so that the mica adhered accurately to the glass surface.

The curved crystal's reflectivity was then measured on the X-ray goniometer system. The reflectivity measurement was done varying the angle of incidence in the direction with no surface curvature of the cylindrical mica crystal. The majority of crystals did not have satisfactory diffraction quality. The best crystals had a typical reflectivity curve as that shown in Figure 12. The 6 mdeg $\left(0.006^{\circ}\right)$ width apparently represents the instrumental contribution for measuring the curved mica crystal, although it is not entirely clear if this instrumental limit could not be closer to the flat crystal measured value of $0.004^{\circ}$. This 6 mdeg $\left(0.006^{\circ}\right)$ reflectivity curve width became the criterion for selecting spectrometer crystals. 
TABLE 4: Calculated germanium 111 crystal properties at $11442.3 \mathrm{eV}$.

\begin{tabular}{lccccc}
\hline & $R_{p}$ & FWHM, mdeg & FWHM, $\mu$ rad & $\begin{array}{c}\text { Integrated reflection } \\
\text { curve, mdeg }\end{array}$ & $\begin{array}{c}\text { Integrated reflection } \\
\text { curve, } \mu \text { rad }\end{array}$ \\
\hline Mixed polarization & 0.724 & 2.743 & 47.87 & 2.297 & 40.06 \\
Measured & 0.27 & 7.5 & 131 & 2.34 & 40.8 \\
\hline
\end{tabular}

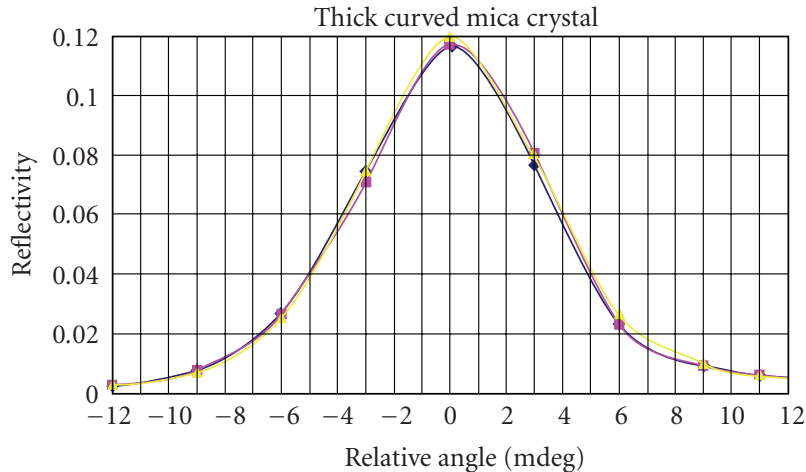

FIGURE 13: Typical best reflectivity curves for thick $(300 \mu \mathrm{m})$ mica crystal from three measurements (shown with three different, closely overlapping, colored curves).

For reference, several curved $300 \mu \mathrm{m}$ thick crystals were measured. Typical reflectivity curves for the best crystals are shown in Figure 13. The FWHM of $0.008^{\circ}$ is larger than the $0.006^{\circ}$ width obtained from the best thin curved crystal measurements. This is consistent with the calculated broadening of a thick mica crystal of 4 mdeg shown in Figure 5.

Germanium was chosen as a backup for the mica crystal for use in the initial testing of the spectrometer. Its reflectivity properties at $11442.3 \mathrm{eV}$ are given in Table 4 . Reflectivity measurements of a $500 \mu \mathrm{m}$ thick germanium crystal bent to a $1.6 \mathrm{~m}$ radius of curvature are given in the last row of the table. The measured reflectivity curve width for the curved germanium crystal is slightly less than that of the thick mica crystal curved to the same radius, and this is consistent with theoretical calculations.

\section{Further Experiments Using an X-ray Source}

NSTec Livermore Operations also has a high energy, open beam X-ray source. It uses a $160 \mathrm{kV}$ source to produce fluorescence from various materials. X-ray beams from $8 \mathrm{keV}$ to $100 \mathrm{keV}$ can be generated. The spectrometer can be aligned and tested using this facility. The $\mathrm{Rb} \mathrm{K}_{\alpha}$ and $\mathrm{Bi} \mathrm{L}_{\alpha}$ spectral lines will be used since they are near the Kr XXXV He like lines.

The spectrometer will be mounted and aligned to the $\mathrm{X}$-ray beam. Image plates and film will be used to turn the alignment and focus the spectrometer. An upgrade to the dual goniometer system is planned for 2010. This includes a high resolution rotator stage and upgraded monochromator crystals.

\section{Conclusion}

The goniometer system of the NSTec Livermore Operations, although limited in resolution, is a good tool for finding satisfactory crystals for hard X-ray spectrometers like the Vertical Johann Spectrometer described in this work. Because of the instrument's construction it is not possible to measure the true crystal resolving power. It is able to differentiate crystal diffraction performance sufficiently to show when the reflectivity curve width is $\Delta \Theta \leq 2.8 \mathrm{mdeg}$ (see Table 3 ) so that $\Delta E \leq 5.5 \mathrm{eV}$ at $13.3 \mathrm{keV}$ (6) and is usable for the Doppler width measurements. The process of selecting suitable mica crystals, curving them accurately to a precision glass substrate, and thinning them to a thickness less than 10 microns is difficult and time consuming. A tool such as the NSTec goniometer system has proved to be invaluable for evaluating the diffraction quality of the crystals needed for this work.

\section{Acknowledgments}

Significant contributions to the success of this work were made by the following colleagues. Jim Emig developed techniques to glue the mica crystal to the flat and curved glass substrates and techniques to cleave the mica. Fletcher Goldin designed a number of mechanical improvements to the alignment procedure to allow us to get the instrument contribution down to the level given in the paper. Ken Jacoby produced much of the reflectivity curve data. This manuscript has been authored by National Security Technologies, LLC, under Contract no. DE-AC52-06NA25946 with the U.S. Department of Energy. The United States Government retains and the publisher, by accepting the article for publication, acknowledges that the United States Government retains a non-exclusive, paid-up, irrevocable, world-wide license to publish or reproduce the published form of this manuscript, or allow others to do so, for United States Government purposes. DOE/NV/25946-839. This work was done under the auspices of the U.S. Department of Energy by Lawrence Livermore National Laboratory under Contract DE-AC52-07NA27344. LLNL-JRNL-420787.

\section{References}

[1] I. Golovkin, R. Mancini, S. Louis et al., "Spectroscopic determination of dynamic plasma gradients in implosion cores," Physical Review Letters, vol. 88, no. 4, Article ID 045002, pp. 450021-450024, 2002.

[2] J. A. Koch, N. Izumi, L. A. Welser et al., "Core temperature and density profile measurements in inertial confinement fusion implosions," High Energy Density Physics, vol. 4, no. 1-2, pp. $1-17,2008$. 
[3] B. Yaakobi, R. Epstein, C. F. Hooper Jr., D. A. Haynes Jr., and Q. Su, "Diagnosis of high-temperature implosions using lowand high-opacity Krypton lines," Journal of X-Ray Science and Technology, vol. 6, no. 2, pp. 172-187, 1996.

[4] B. Yaakobi, F. J. Marshall, and R. Epstein, "High temperature of laser-compressed shells measured with $\mathrm{Kr} 34+$ and $\mathrm{Kr} 35+$ X-ray lines," Physical Review E, vol. 54, no. 5, pp. 5848-5850, 1996.

[5] M. Bitter, S. Von Goeler, R. Horton et al., "Dopplerbroadening measurements of $\mathrm{x}$-ray lines for determination of the ion temperature in tokamak plasmas," Physical Review Letters, vol. 42, no. 5, pp. 304-307, 1979.

[6] O. Renner, T. Missalla, P. Sondhauss et al., "High-luminosity, high-resolution, $\mathrm{x}$-ray spectroscopy of laser-produced plasma by vertical-geometry Johann spectrometer," Review of Scientific Instruments, vol. 68, no. 6, pp. 2393-2403, 1997.

[7] O. Renner, S. G. Podorov, O. Wehrhan, and E. Förster, "Vertical dispersion Johann $\mathrm{x}$-ray spectrometer with asymmetrically cut crystal," Review of Scientific Instruments, vol. 75, no. 11, pp. 4569-4577, 2004.

[8] M. Kopecký, "A modified scheme of Johann spectrograph," Review of Scientific Instruments, vol. 66, no. 10, pp. 4921-4924, 1995.

[9] I. Uschmann, E. Forster, K. Bell, and G. Holzer, "X-ray reflection properties of elastically bent perfect crystals in bragg geometry," Journal of Applied Crystallography, vol. 26, pp. 405412, 1993.

[10] I. Uschmann, A. C. Malgrange, and E. Forster, "Measurement of a bent crystal rocking curve with oscillations," Journal of Applied Crystallography, vol. 30, pp. 1150-1151, 1997.

[11] D. Taupin, "Théorie dynamique de la diffraction des rayons $\mathrm{X}$ par les cristaux déformés," Bulletin de la Société Française de Minéralogie et de Cristallographie, vol. 84, pp. 469-511, 1964.

[12] S. G. Podorov and E. Förster, "Theory of X-ray diffraction on asymmetrically cut and bent crystals," Physica Status Solidi A, vol. 220, no. 2, pp. 829-836, 2000.

[13] S. G. Podorov, "X-Ray for Windows," www.podorov.de/ programs.htm.

[14] S. Tolansy, Multiple Beam Interferometry of Surfaces and Films, Dover, New York, NY, USA, 1970.

[15] S. Stepanov, "X0h on the Web," http://sergey.gmca.aps.anl .gov/x0h.html. 